\title{
Analysis of the Asteroseismological Data of Six $\delta$ Scuti Stars in the Open Cluster NGC 6134
}

\author{
Nathalie Audard, Hans Kjeldsen, Søren Frandsen \\ Institut for Fysik og Astronomi, Aarhus Universitet, DK 8000 Aarhus \\ $C$, Denmark
}

\begin{abstract}
Six $\delta$ Scuti stars have been detected in the open cluster NGC 6134 (Frandsen et al. 1995). A first mode identification attempt shows that several radial modes of radial order $n=0,1,2$ or 3 might have been observed. We may also be dealing with $g$-modes and/or modes split by rotation.
\end{abstract}

\section{Theoretical isochrones}

We use the photometric data obtained by Frandsen et al. (1995).

Models are computed with the CESAM code (Morel, 1993). It turns out that isochrones constructed with convective-core overshooting over 0.2 pressure scale height provide a better fit than those derived from standard models.

\section{Asteroseismology of stars in the open cluster NGC 6134}

Table 1 gives the ID number of the variables, their observed frequencies and periods $P$, and the corresponding pulsation constants $Q=P \sqrt{M / R^{3}}$ (where $M$ is the stellar mass and $R$ the radius), as well as the amplitude of the modes (columns 1 to 5 respectively).

We compare these $Q$ values to those provided by Stellingwerf (1979): Stellingwerf's model number 2.1 for the star 9 , number 4.2 for the stars 87,159 and 397 , number 1.1 for the star 161, and number 2.2 for the star 348. Table 1 shows the resulting possible identification (last column).

The first four frequencies of star 9 are so close that they could be modes split by rotation. But since the $Q$ values for these modes are larger than those of Stellingwerf's fundamental mode, they may also be $g$-modes. For the same reason, the lower frequencies of the stars 159 and 348 may also be $g$-modes.

\section{Conclusion}

Our main results are the following:

- Convective-core overshooting is needed in order to make isochrones which fit the observations.

- A mode identification attempt shows that several of the observed oscillations may be fundamental, first, second and third radial modes. 
- Some oscillations having very close frequencies could be $g$-modes and/or modes split by rotation. The observation of $g$-modes would be of great interest for studying the deep stellar interior and the importance of convective-core overshooting.

However, we must keep in mind that the $Q$ values derived from the observations are subject to uncertainties in the calculation of the effective temperature, mass and radius. Moreover, rotation is not taken into account in our computations, and may lead to a different identification of the modes.

Table 1. Asteroseismological data of the six $\delta$ Scuti stars in NGC 6134: ID numbers, frequencies, periods, pulsation constants $Q$, amplitudes and possible mode identification.

\begin{tabular}{|c|c|c|c|c|c|}
\hline ID & $\begin{array}{c}\text { Frequency } \\
(\mu \mathrm{Hz})\end{array}$ & $\begin{array}{l}\text { Period } \\
\text { (days) }\end{array}$ & $\begin{array}{c}\bar{Q} \\
\text { (days) }\end{array}$ & $\begin{array}{l}\text { Amplitude } \\
\text { (mmag) }\end{array}$ & $\begin{array}{c}\text { Possible mode } \\
\text { identification }(n, \ell)\end{array}$ \\
\hline \multirow[t]{5}{*}{9} & 59.3 & 0.1958 & 0.0395 & 6.4 & $g$-mode \\
\hline & 61.2 & 0.1891 & 0.0383 & 6.7 & $g$-mode \\
\hline & 63.5 & 0.1823 & 0.0369 & 9.7 & $g$-mode \\
\hline & 65.6 & 0.1764 & 0.0357 & 8.1 & $g$-mode \\
\hline & 89.8 & 0.1289 & 0.0261 & 4.3 & $(2,0)$ \\
\hline \multirow[t]{3}{*}{87} & 162.4 & 0.0713 & 0.0319 & 0.8 & \\
\hline & 200.5 & 0.0577 & 0.0258 & 2.0 & $(2,0)$ \\
\hline & 220.5 & 0.0525 & 0.0235 & 0.8 & \\
\hline \multirow[t]{3}{*}{159} & 116.1 & 0.0997 & 0.0384 & 4.5 & $g$-mode \\
\hline & 139.3 & 0.0839 & 0.0320 & 5.9 & \\
\hline & 244.8 & 0.0478 & 0.0182 & 4.1 & $(4,0)$ \\
\hline 161 & 87.3 & 0.1326 & 0.0224 & 6.2 & \\
\hline \multirow[t]{2}{*}{348} & 76.4 & 0.1515 & 0.0363 & 4.2 & $g$-mode \\
\hline & 133.5 & 0.0867 & 0.0208 & 6.2 & $(3,0)$ \\
\hline \multirow[t]{2}{*}{397} & 168.1 & 0.0689 & 0.0317 & 1.7 & \\
\hline & 324.3 & 0.0357 & 0.0164 & 1.6 & \\
\hline
\end{tabular}

\section{References}

Frandsen, S., Balona, L.A., Viskum, M., Koen, C., \& Kjeldsen, H. 1995, "Multisite CCD Photometry of $\delta$ Scuti stars in the open cluster NGC 6134 (I STACC Campaign)", to be submitted to A\&A

Morel, P. 1993, in "Inside the Stars", Weiss W.W., Baglin A. (eds), ASP Conf. Ser., vol. 40 , p. 445

Stellingwerf, R.F. 1979, ApJ, 227, 935 